\title{
Individual Barkhausen Pulses of Ferroelastic Nanodomains
}

\author{
Reinis Ignatans $\odot$, Dragan Damjanovic $\odot$, and Vasiliki Tileli®* \\ Institute of Materials, École polytechnique fédérale de Lausanne, Station 12, 1015 Lausanne, Switzerland
}

(Received 1 March 2021; accepted 3 September 2021; published 12 October 2021)

\begin{abstract}
Ferroelectric materials, upon electric field biasing, display polarization discontinuities known as Barkhausen jumps, a subclass of a more general phenomenon known as crackling noise. Herein, we follow and visualize in real time the motion of single $90^{\circ}$ needle domains induced by an electric field applied in the polarization direction of the prototypical ferroelectric $\mathrm{BaTiO}_{3}$, inside a transmission electron microscope. The nature of motion and periodicity of the Barkhausen pulses leads to distinctive interactions between domains forming a herringbone pattern. Remarkably, the tips of the domains do not come into contact with the body of the perpendicular domain, suggesting the presence of strong electromechanical fields around the tips of the needle domains. Additionally, interactions of the domains with the lattice result in relatively free movement of the domain walls through the dielectric medium, indicating that their motion-related activation energy depends only on weak Peierls-like potentials. Control over the kinetics of ferroelastic domain wall motion can lead to novel nanoelectronic devices pertinent to computing and data storage applications.
\end{abstract}

DOI: 10.1103/PhysRevLett.127.167601

Polarization switching in ferroelectric crystals is akin to first order phase transition induced by an applied electric field [1]. The reorientation of the domains during switching is a response to assume the equilibrium conditions that minimize the free energies of domain configuration. The motion toward the equilibrium state is hindered by potential barriers that lead to nonmonotonous polarization change discontinuities referred to as Barkhausen effect [2-6]. The accumulated probing of this effect describing the motion of many domain walls is linked to the theory of avalanches and the noise associated with it $[7,8]$. More generally, crackling noise seems to be a fundamental aspect of nature and "crackles" occur in vastly different systems and processes $[9,10]$.

In ceramics [5] and single crystalline $\mathrm{BaTiO}_{3}[3,11]$, the origins of the Barkhausen effect are placed on nucleation of spikelike domains, pinning of domain walls due to defects in the lattice [2,6], domain coalescence [12], and transition of the needlelike to parallelepiped domains [11]. Most electrical studies of Barkhausen jumps attempt to resolve single events from macroscopic properties of ferroelectrics (such as switching current or charge) and the different methodologies used can reveal different origins of the pulses. This is associated with variations in measurement timescales, boundary conditions, and physical dimensions

Published by the American Physical Society under the terms of the Creative Commons Attribution 4.0 International license. Further distribution of this work must maintain attribution to the author(s) and the published article's title, journal citation, and DOI. of the material. Typically, to characterize these effects, in situ optical microscopy experiments are performed [13]. However, optical measurements are diffraction limited and fine polar regions, such as the tips of needle domains, are difficult to probe in detail. At nanoscale dimensions traditional electrical measurements become exponentially more difficult due to miniscule charges associated with individual switching events. For example, in situ transmission electron microscopy (TEM) experiments have been previously performed in $\mathrm{BaTiO}_{3}$ single crystals to enhance the spatial resolution of the domain dynamics [14-17]. In general, kinetically controlled studies of nanodomain switching and the way their interaction affects their motion in TEM are still limited due to the overall geometric confinement of the specimen, the electric field uniformity in the probed area, and the effects of the electron beam irradiation.

In the following, we stabilize $90^{\circ}$ ferroelastic nanodomains and track their movement inside the TEM by applying a well-oriented electric field. We locally probe the Barkhausen jumps for different potential barriers and at different electric field frequencies. To achieve this, we prepared lamellae specimen along the pseudocubic $[100]_{\mathrm{PC}}$ orientation from a single crystal $\mathrm{BaTiO}_{3}$ and placed it on a microelectromechanical system (MEMS) chip patterned with six electrodes, four used for temperature control and two for electric field biasing. The geometry of the device (Fig. S1 and Materials and Methods in Supplemental Material [18]) permits application of homogeneous electric field across the probed sample area [19].

Figure 1(a) depicts the domain structure at nominal temperature of $130^{\circ} \mathrm{C}$ (see Supplemental Material [18] for 
(a)

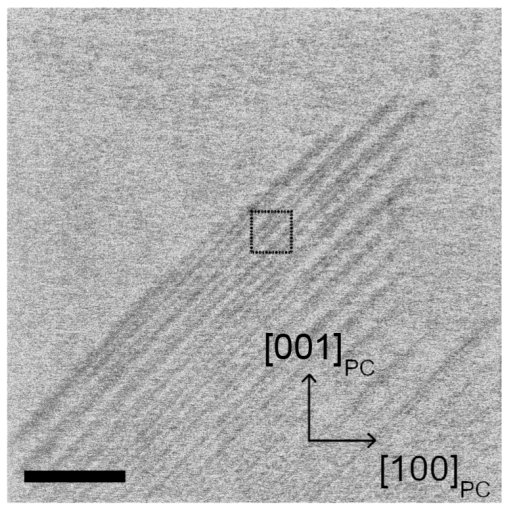

(b)

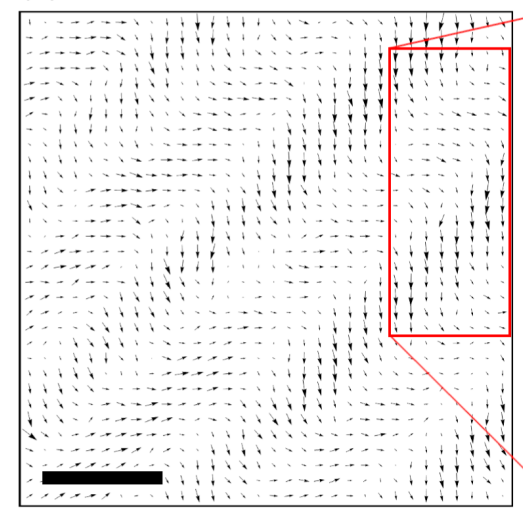

(c)

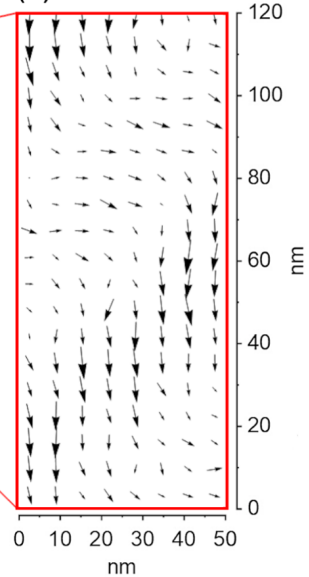

FIG. 1. (a) Domain structure at $130{ }^{\circ} \mathrm{C}$ showing periodic ferroelastic $90^{\circ}$ needle domains with polarization direction along $[100]_{\mathrm{PC}}$ (scale bar is $500 \mathrm{~nm}$ ). (b) DPC image of the black dotted square area in (a) (scale bar is $50 \mathrm{~nm}$ ). The arrows represent the polarization direction of the needle and parent domains. (c) Close-up of polarization vectors of the ferroelastic nanodomains.

details on the temperature measurement), which consists of periodic $90^{\circ}$ ferroelastic needle domain groups (the full heating profile and image series is shown in Supplemental Material, Video S1 [18]). We note that similar domain evolution is seen in a free standing lamella (Fig. S2) and, hence, this behavior is not associated with the geometry of the biasing device. To characterize the ferroelastic domain structure, differential phase contrast (DPC) imaging [20] was employed. The DPC signal is directly proportional to the magnitude and direction of the local polarization [20]. Figure 1(b) represents the local profile of polarization directions encoded by arrows from the square region shown in Fig. 1(a). The size of the arrows is proportional to the magnitude of the polarization, however, absolute values are difficult to report since minor misalignment greatly affects the DPC results, which is the reason why the magnitude of the arrows in the horizontal and vertical directions are not exactly the same. Nevertheless, Fig. 1(c) illustrates that the $90^{\circ}$ domain walls in tetragonal $\mathrm{BaTiO}_{3}$ align with the $[110]_{\mathrm{PC}}$ direction [1], as expected from crystallographic symmetry laws. The measured average domain width $w$ is $33 \mathrm{~nm}$. The domain walls are less than $7 \mathrm{~nm}$ thick and they are associated with dark contrast (i.e., high angle scattering) in bright-field scanning TEM (BF STEM) images. The appearance of the periodic domain structure in thin films results from the proportional relationship between the square of $w$ with the slab's thickness $d$ [21-25].

At $130^{\circ} \mathrm{C}$, we applied a triangular waveform voltage (see Supplemental Material, Methods and Fig. S3 [18]) and followed the response of several $90^{\circ}$, ferroelastic, $a$-type, needlelike domains, Fig. 2(a). The full sequence of BF STEM images of a cyclic measurement for an applied bias of $\pm 3.5 \mathrm{~V}$ can be seen in Supplemental Material, Video S2 [18]. The collective statistical behavior of the system has also been analyzed by machine learning algorithms and is reported elsewhere [26]. Figures 2(b) and 2(c) depict the domain length as a function of applied potential for two needle domains. These plots highly resemble traditional polarization-electric field (PE) loops, however, while conventionally measured PE loops show macroscopically averaged behavior, here, we follow the response locally on a single domain basis. We have observed two distinct manifestations of the needle domain response on the electric field. When the vertex of a needle domain is located further away from the perpendicular domain walls [Fig. 2(b)], the measured domain length vs applied electric field loop is smeared, with reduced hysteresis, and the process is lattice-defect mediated [27,28]. However, when the needle domain's vertex is located close to the perpendicular domain's wall [Fig. 2(c)] the shape of the measured loop is sharp and squarelike showing pronounced hysteresis. Therefore, this domain-domain interaction mediated process seems to involve strong local strain and depolarizing fields leading to large hysteresis, unlike the lattice-defect mediated process. Measurements of the motion of perpendicular needle domains show similar behavior (Supplemental Material, Fig. S4 [18]).

Closer investigation of the loop in Fig. 2(c) shows clear steplike features around, for example, 1 and $2 \mathrm{~V}$, whereas for needle domains whose vertices lie further away from the perpendicular needle domain walls, steplike features are less pronounced. Thus, needle domains whose vertices are close to the perpendicular domain walls experience distinct nonmonotonous movement during biasing [5]. These Barkhausen jumps occur when the perpendicular needle domains have contracted. In practice, needle domains do not interact directly with perpendicular domains (i.e., they are not in direct contact with them), but rather interaction of needle domains is mediated through a large parent domain [the background gray domain in the images of Fig. 2(a)]. In general, the equilibrium position of the needle domains between Barkhausen jumps is associated with electric and mechanical compatibility [29-31]. Two possibilities can be 
(a)
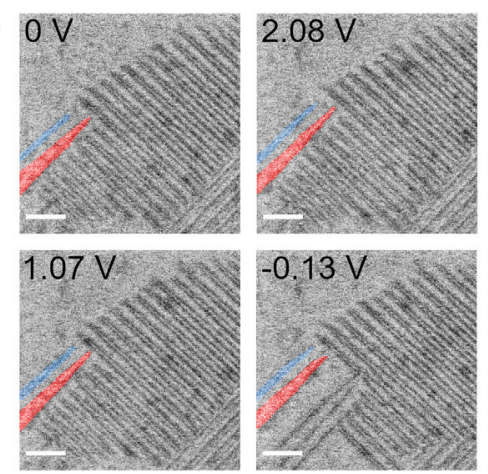
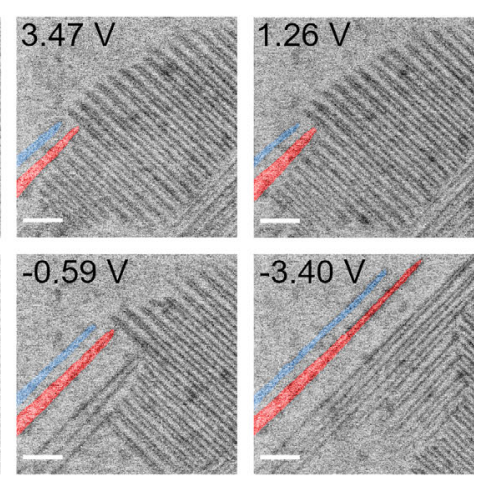

(b) (c)

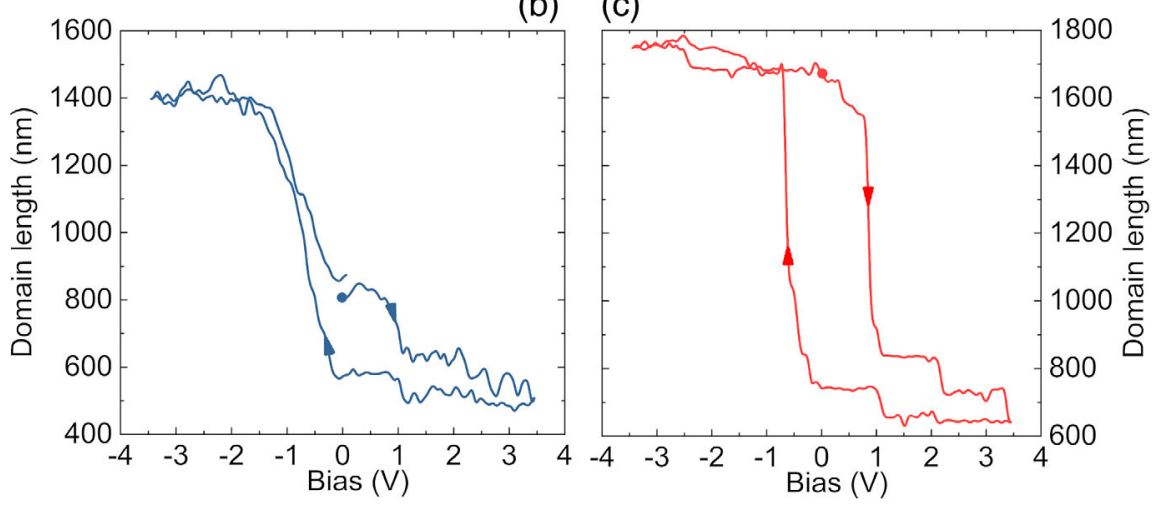

FIG. 2. (a) $90^{\circ}$ domain structure evolution during biasing recorded at $130^{\circ} \mathrm{C}$. The measured domains are overlaid with red and blue false color. The electric field is applied along the needle domains polarization direction, i.e. [100 $]_{\mathrm{PC}}$. Scale bar is $300 \mathrm{~nm}$. (b) Domain length as a function of applied potential plot of a domain with weak interaction with perpendicular domain walls [blue false color in (a)]. (c) Similar loop of a domain that interacts strongly with perpendicular domain walls [red false color in (a)]. The starting point of the loops is marked with a dot and the loop rotation direction is marked with arrows.

distinguished, the first one, where the needle domain's vertex terminates next to the perpendicular needle domain's body, and the second, in which equilibrium is achieved when two or more strongly charged needle domain vertices come in contact [29]. The observed Barkhausen jumps occur among these equilibria. We note that experiments for an increasing maximum range of the applied bias were additionally performed and similar domain behavior was observed (Supplemental Material, Videos S3 to S8 [18]).

To study the time dependence of the needle domain response, similar experiments were carried out with a less steep voltage ramp $(0.0034 \mathrm{~V} / \mathrm{s})$ corresponding to the ultralow frequency of $0.24 \mathrm{mHz}$. The hysteresis loop can be seen in Supplemental Material, Fig. S5, and the full sequence is shown in Video S9 [18]. The overall domain length vs applied voltage loop is steep and squarelike and it is similar to the one in Fig. 2(c) that represents strong domain-domain interaction. Again, Barkhausen jump events associated with interacting needle domain equilibrium positions were recorded.

To compare the response of the processes leading to characteristically different domain wall motion, their length is plotted as a function of time, Fig. 3(a). Both time axes in the plot are rescaled to match the start and the end of the bias cycle. Domains which experience strong mutual interaction (red and green squares), exhibit distinctive step-like Barkhausen jumps (marked in the Fig. 3 with green and red arrows), whereas domain growth via latticemediated defect mechanism is considerably smoother (blue squares). The key difference observed during the low frequency measurement is the appearance of the domain relaxation events with a time constant of roughly three to five minutes. For example, one such event can be seen in Fig. 3(a) between 2500 and $2900 \mathrm{~s}$. The measured domain first decreases in length upon applied negative voltage, then it slowly increases in length (marked with gray horizontal line), and eventually exhibits a Barkhausen jump around $2900 \mathrm{~s}$, before shrinking further. After this jump, the domain again slightly increases in length, showing similar behavior as before. Interestingly, at around 3000-3300 s, when the negative potential slowly returns to zero volts, the domain annihilates completely (marked with the dashed green arrow). In short, when the domain experiences large applied bias, it stays intact, but on the decreasing field it slowly decreases in size and disappears, which again indicates a relaxation event with a time constant of several minutes. The observed sluggish relaxation process can be attributed to dielectric and elastic viscosity [2,32]. According to observations from electrical measurements, the domain structure can relax up to several hours after 

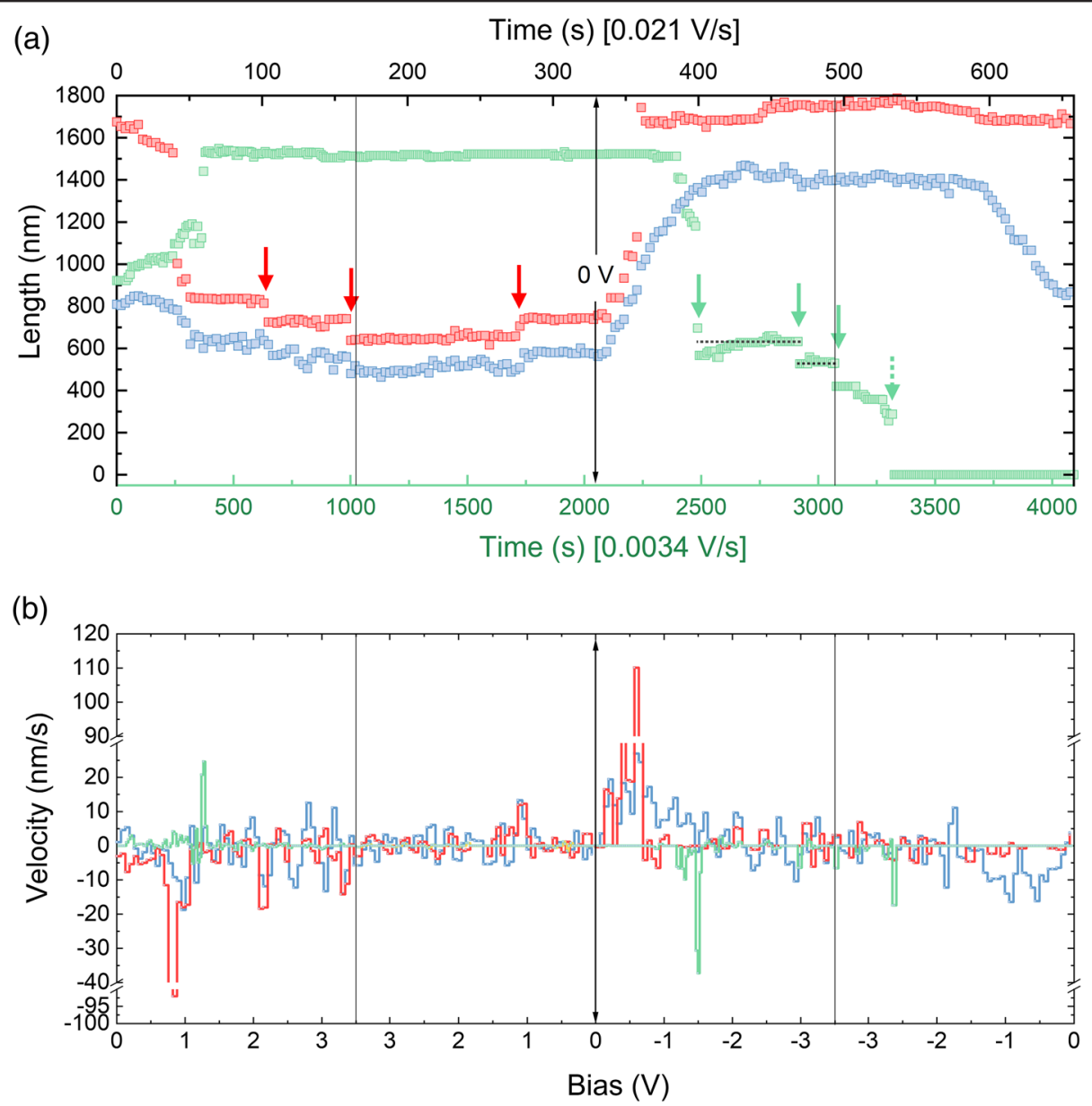

FIG. 3. (a) Domain length plotted as a function of time. The measurements of the two domains [red and blue of Fig. 2(a)] correspond to the top black $x$ axis (performed at $0.021 \mathrm{~V} / \mathrm{s}$ ) whereas the green squares and bottom $x$ axis correspond to the slow domain response in Supplemental Material, Fig. S5 (performed at 0.0034 V/s) [18]. Red and green vertical arrows indicate Barkhausen events. The dashed, green arrow represents the point of annihilation of the domain. Gray dotted horizontal lines represent domain length after relaxation. The domain response of the ultralow frequency measurements is reversed, due to the spontaneous polarization direction upon domain nucleation. (b) Domain tip velocity with respect to applied bias voltage [color coding is the same as in (a)]. Time and bias $x$ axes on the two plots are scaled in such way that they correspond to each other on the basis of the field waveform.

poling. We may therefore be witnessing an individual event responsible for aging and creep in ferroelectric materials [33-35]. Finally, when the lower frequency domain motion is compared to higher frequency ones, we notice a time delay of the switching process when the potential is brought back to zero. During ultralow frequency measurements, the domain remains at the same position for a significant time before it eventually switches.

By numerically differentiating the domain length vs time data [Fig. 3(a)], the speed of the needle domains can be determined. Figure 3(b) shows the velocity as a function of applied voltage for the three distinct domains. Essentially, the speed is proportional to the local switching current (i.e., $i \sim \Delta P / \Delta t)$. By comparing the lattice-mediated growth (blue line) with the domain-domain interaction (red and green lines), it is determined that the domain-tip velocity for the lattice-mediated domain is more smeared out (i.e., the vertex is almost always moving), whereas the tip velocity of domains that interact with each other is accompanied with sharp spikes and, at most times, the velocity is close to zero. This indicates that the potential well is deeper for domain-domain associated pinning as compared to pinning due to the defects within the lattice $[36,37]$. Additionally, in the case of the low frequency measurement (green line), the velocity spikes can be seen in the deep negative electric field region representing Barkhausen jumps due to the simultaneous effects of domain-domain interaction and slow relaxation processes.

Overall, the observed evolution and motion of ferroelectric single needle domains induced by the electric field applied in the polar direction is characteristic of a forward domain growth process [38]. However, we have previously shown that such movements follow Rayleigh-like behavior [19] and, therefore, pinning mechanisms typically studied for lateral domain wall movements are also applicable in this high field regime. Further, we discuss the dynamic 


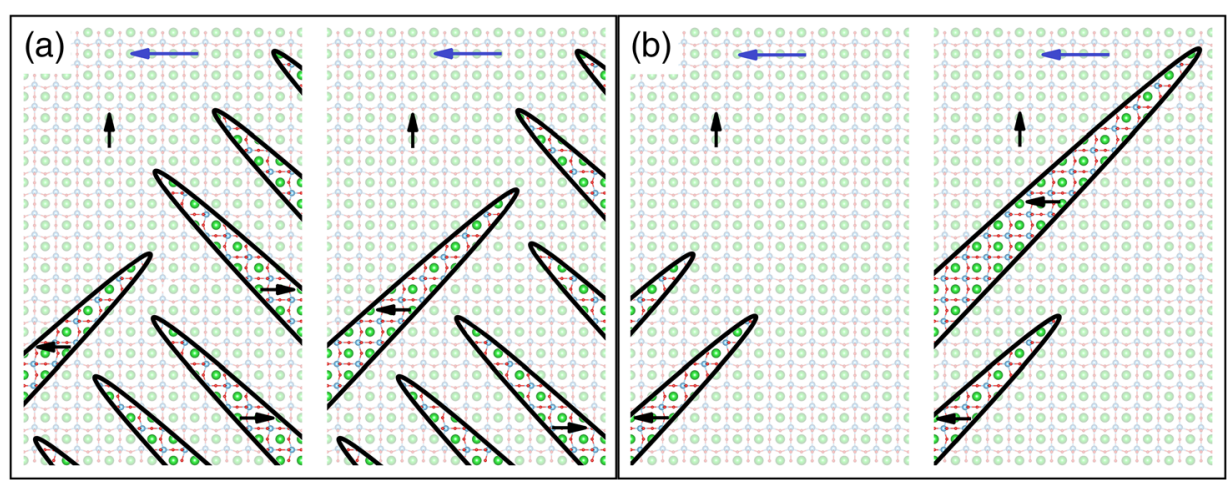

FIG. 4. (a) Needle domain configuration in a metastable electromechanical equilibrium before a Barkhausen event and new equilibrium after the application of external electric field in the polar direction of the needle domains. (b) Configuration of two needle domains located far from perpendicular domain walls. The electric field direction is depicted with blue arrows and the polarization directions in all domains are shown with black arrows.

behavior of the domains on the basis of the schematics in Fig. 4. Our results show that when a herringbone domain pattern is adopted, a separation between perpendicular domains that never come into contact with each other is probed [Fig. 4(a)]. Images associated with this phenomenon are shown and inspected in Supplemental Material, Fig. S6 [18]. Moreover, the depolarizing and strain fields at the needle tip form a large potential barrier that manifests as well-defined Barkhausen jumps. The positions of these jumps are associated with the annihilation of the periodic, perpendicular domains. The motion of the domains through potentials determined by domain-domain pinning interactions leads to hysteretic behavior. A different mechanism is encountered when parallel needle domains are free to move within the lattice [Fig. 4(b)]. In this case, the Barkhausen pulses are rare events, and are most likely dominated by Peierls potential barriers due to lattice potential and point defects in the nonperfect crystal $[36,37]$. The associated shallow potential barrier does not affect perpendicular domain-domain motion but is dominating the jump frequency during the motion of single domains across the lattice. Therefore, at the moderate electric field and frequencies used herein, Barkhausen jumps mostly originate from domain-domain interactions without domains actually touching each other and much less from interaction of domains with point defects.

In conclusion, we have demonstrated individual ferroelastic needle domain response on the applied electric field leading to strong pinning and consequential Barkhausen jumps in between domain structure metastable equilibria. The mechanisms of domain-domain interactions among noncontacting domains remain largely unexplored. Our experimental study shows that major domain pinning and restriction to movement in a thin single crystal $\mathrm{BaTiO}_{3}$ ferroelectric is predominantly associated with contactless mutual domains interaction and less with Peierls potential pinning arising from the lattice in an undoped crystal. The shape of the measured domain length-electric bias loops hints that domain-domain interaction may be dominant in the expression of the materials properties macroscopically. Individual local relaxation events leading to aging and creep have also been observed. Such insights gained from local measurements performed in a thin ferroelectric slab, can be relevant for modern technologies related to local and global polarization switching.

The work was supported by the Swiss National Science Foundation (SNSF) under Grant No. 200021_175711. D. D. acknowledges support by the ONR Global (Grants No. N62909-18-1-2078 and No. N62909-20-1-2083).

*Correspondending author. vasiliki.tileli@epfl.ch

[1] A. K. Tagantsev, L. E. Cross, and J. Fousek, Domains in Ferroic Crystals and Thin Films (Springer New York, 2010), http://link.springer.com/10.1007/978-1-4419-1417-0).

[2] V. M. Rudyak, The Barkhausen effect, Sov. Phys. Usp. 13, 461 (1971).

[3] A. G. Chynoweth, Barkhausen pulses in barium titanate, Phys. Rev. 110, 1316 (1958).

[4] W. J. Merz, Domain formation and domain wall motions in ferroelectric $\mathrm{BaTiO}_{3}$ single crystals, Phys. Rev. 95, 690 (1954).

[5] C. D. Tan, C. Flannigan, J. Gardner, F. D. Morrison, E. K. H. Salje, and J.F. Scott, Electrical studies of Barkhausen switching noise in ferroelectric PZT: Critical exponents and temperature dependence, Phys. Rev. Mater. 3, 034402 (2019).

[6] C. Flannigan, C. D. Tan, and J. F. Scott, Electrical studies of Barkhausen switching noise in ferroelectric lead zirconate titanate (PZT) and $\mathrm{BaTiO}_{3}$ : Critical exponents and temperature-dependence, J. Phys. Condens. Matter 32, 055403 (2020).

[7] Y. Xu, Y. Zhou, D. Xue, X. Ding, J. Sun, and E. K. H. Salje, Anisotropic avalanche dynamics during ferroelectric switching in $\mathrm{BaTiO}_{3}$ and $0.7 \mathrm{~Pb}\left(\mathrm{Mg}_{2 / 3} \mathrm{Nb}_{1 / 3}\right) \mathrm{O}_{3}-0.3 \mathrm{PbTiO}_{3}$, Appl. Phys. Lett. 117, 172901 (2020). 
[8] E. K. H. Salje, D. Xue, X. Ding, K. A. Dahmen, and J. F. Scott, Ferroelectric switching and scale invariant avalanches in $\mathrm{BaTiO}_{3}$, Phys. Rev. Mater. 3, 014415 (2019).

[9] E. K. H. Salje and K. A. Dahmen, Crackling noise in disordered materials, Annu. Rev. Condens. Matter Phys. 5, 233 (2014).

[10] G. F. Nataf, M. Guennou, J. M. Gregg, D. Meier, J. Hlinka, E. K. H. Salje, and J. Kreisel, Domain-wall engineering and topological defects in ferroelectric and ferroelastic materials, Nat. Rev. Phys. 2, 634 (2020).

[11] B. BŘezina, J. Fousek, and A. Glanc, Barkhausen pulses in $\mathrm{BaTiO}_{3}$ connected with $90^{\circ}$ switching processes, Czech. J. Phys. 11, 595 (1961).

[12] R. C. Miller, On the origin of Barkhausen pulses in $\mathrm{BaTiO}_{3}$, J. Phys. Chem. Solids 17, 93 (1960).

[13] P. Potnis and J. Huber, In-situ observation of needle domain evolution in barium titanate single crystals, J. Eur. Ceram. Soc. 33, 327 (2013).

[14] E. Snoeck, L. Normand, A. Thorel, and C. Roucau, Electron microscopy study of ferroelastic and ferroelectric domain wall motions induced by the in situ application of an electric field in $\mathrm{BaTiO}_{3}$, Phase Transitions 46, 77 (1994).

[15] M. He, M. Wang, and Z. Zhang, Electric-field-induced domain intersection in $\mathrm{BaTiO}_{3}$ single crystal, Jpn. J. Appl. Phys. 56, 031501 (2017).

[16] X. Y. Qi, H. H. Liu, and X. F. Duan, In situ transmission electron microscopy study of electric-field-induced $90^{\circ}$ domain switching in $\mathrm{BaTiO}_{3}$ single crystals, Appl. Phys. Lett. 89, 092908 (2006).

[17] Z. H. Zhang, X. Y. Qi, and X. F. Duan, Two-step evolution mechanism of multi-domains in $\mathrm{BaTiO}_{3}$ single crystal investigated by in situ transmission electron microscopy, Scr. Mater. 58, 441 (2008).

[18] See Supplemental Material at http://link.aps.org/supplemental/ 10.1103/PhysRevLett.127.167601 for details on materials and methods, additional experimental results included in Figs. S1-S6, and captions for Videos S1-S9

[19] R. Ignatans, D. Damjanovic, and V. Tileli, Local "hard" and "soft" pinning of $180^{\circ}$ domain walls in $\mathrm{BaTiO}_{3}$ probed by in situ transmission electron microscopy, Phys. Rev. Mater. 4, 104403 (2020).

[20] B. Haas, J.-L. Rouvière, V. Boureau, R. Berthier, and D. Cooper, Direct comparison of off-axis holography and differential phase contrast for the mapping of electric fields in semiconductors by transmission electron microscopy, Ultramicroscopy 198, 58 (2019).

[21] C. Kittel, Theory of the structure of ferromagnetic domains in films and small particles, Phys. Rev. 70, 965 (1946).

[22] A. L. Roitburd, Equilibrium structure of epitaxial layers, Phys. Status Solidi 37, 329 (1976).

[23] A. Schilling, T. B. Adams, R. M. Bowman, J. M. Gregg, G. Catalan, and J. F. Scott, Scaling of domain periodicity with thickness measured in $\mathrm{BaTiO}_{3}$ single crystal lamellae and comparison with other ferroics, Phys. Rev. B 74, 024115 (2006).

[24] J. S. Speck and W. Pompe, Domain configurations due to multiple misfit relaxation mechanisms in epitaxial ferroelectric thin films. I. Theory, J. Appl. Phys. 76, 466 (1994).

[25] J. S. Speck, A. Seifert, W. Pompe, and R. Ramesh, Domain configurations due to multiple misfit relaxation mechanisms in epitaxial ferroelectric thin films. II. Experimental verification and implications, J. Appl. Phys. 76, 477 (1994).

[26] R. Ignatans, M. Ziatdinov, R. Vasudevan, M. Valleti, V. Tileli, and S. V. Kalinin, Latent mechanisms of polarization switching from in situ electron microscopy observations, arXiv:2011.11869.

[27] D. Damjanovic, Ferroelectric, dielectric and piezoelectric properties of ferroelectric thin films and ceramics, Rep. Prog. Phys. 61, 1267 (1998).

[28] L. Mitoseriu, V. Tura, D. Ricinschi, and C. Harnagea, Grain size dependence of the Rayleigh coefficients in barium titanate ceramics, Ferroelectrics 240, 1317 (2000).

[29] D. Sui and J. E. Huber, Modelling and interaction of needle domains in barium titanate single crystals, Eur. J. Mech. 80, 103919 (2020).

[30] E. K. H. Salje and Y. Ishibashi, Mesoscopic structures in ferroelastic crystals: Needle twins and right-angled domains, J. Phys. Condens. Matter 8, 8477 (1996).

[31] S. Puchberger, V. Soprunyuk, W. Schranz, A. Tröster, K. Roleder, A. Majchrowski, M. A. Carpenter, and E. K. H. Salje, The noise of many needles: Jerky domain wall propagation in $\mathrm{PbZrO}_{3}$ and $\mathrm{LaAlO}_{3}$, APL Mater. 5, 046102 (2017).

[32] R. J. Harrison, S. A. T. Redfern, and E. K. H. Salje, Dynamical excitation and anelastic relaxation of ferroelastic domain walls in $\mathrm{LaAlO}_{3}$, Phys. Rev. B 69, 144101 (2004).

[33] B. Ziegler, K. Martens, T. Giamarchi, and P. Paruch, Domain Wall Roughness in Stripe Phase $\mathrm{BiFeO}_{3}$ Thin Films, Phys. Rev. Lett. 111, 247604 (2013).

[34] P. Paruch, T. Giamarchi, and J.-M. Triscone, Domain Wall Roughness in Epitaxial Ferroelectric $\mathrm{PbZr}_{0.2} \mathrm{Ti}_{0.8} \mathrm{O}_{3}$ Thin Films, Phys. Rev. Lett. 94, 197601 (2005).

[35] T. Tybell, P. Paruch, T. Giamarchi, and J.-M. Triscone, Domain Wall Creep in Epitaxial Ferroelectric $\mathrm{Pb}\left(\mathrm{Zr}_{0.2} \mathrm{Ti}_{0.8}\right) \mathrm{O}_{3}$ Thin Films, Phys. Rev. Lett. 89, 097601 (2002).

[36] K. S. Novoselov, A. K. Geim, S. V. Dubonos, E. W. Hill, and I. V. Grigorieva, Subatomic movements of a domain wall in the Peierls potential, Nature (London) 426, 812 (2003).

[37] H. Ma, W. J. Kim, J. S. Horwitz, S. W. Kirchoefer, and J. Levy, Lattice-Scale Domain Wall Dynamics in Ferroelectrics, Phys. Rev. Lett. 91, 217601 (2003).

[38] V. Y. Shur and A. R. Akhmatkhanov, Domain shape instabilities and dendrite domain growth in uniaxial ferroelectrics, Phil. Trans. R. Soc. A 376, 20170204 (2018). 